\title{
Vector-mediated Tum-5 expression in neovascular endothelial cells for treating hepatocellular carcinoma
}

\author{
CHUN LI, XINGANG GUAN, BOQIAN SUN, MINGYAO MA, PENG WANG and XIAODONG GAI \\ Department of Pathology, School of Basic Medical Sciences, Beihua University, Jilin City, Jilin 132013, P.R. China
}

Received September 3, 2015; Accepted October 18, 2016

DOI: $10.3892 / \mathrm{etm} .2017 .4127$

\begin{abstract}
Hypervascular hepatocellular carcinoma (HCC) is one of the leading causes of cancer-associated mortality. Angiogenesis is an important contributor to HCC progression and metastasis; therefore, inhibiting angiogenesis may be an effective method of treating HCC. Tumstatin is a novel type of efficient endogenous vascular endothelial cell growth inhibiting factor. The anti-angiogenic activity of tumstatin is localized to the 54-132 amino acid region (Tum-5). In a previous study performed by our group, the gene fragment encoding Tum-5 was cloned and inserted into a pLXSN retroviral vector. In the present study, the anti-angiogenic effects of Tum-5 and the antitumor effects exerted by the pLXSN-Tum-5 vector in vivo were investigated. The results demonstrated that pLXSN-Tum-5 significantly inhibited the growth of human umbilical vein endothelial cells compared with pLXSN, but had no obvious effect on HepG2 cell growth. Moreover, the antitumor and anti-angiogenic activity of Tum-5 was examined in vivo using a xenograft of $\mathrm{H} 22 \mathrm{HCC}$ cells. The results indicated that pLXSN-Tum-5 significantly inhibited tumor growth following 5 injections over 10 days. The size and weight of tumors in the pLXSN-Tum-5 group were lower than those in the saline and pLXSN groups. Furthermore, immunohistochemical analysis with CD31 antibodies indicated that the average microvessel density in the pLXSN-Tum-5 group were significantly lower than that in the saline and pLXSN groups. These results suggested that Tum-5 exerts its antitumor activity by suppressing vascular endothelial cells. The gene fragment of Tum-5 may be developed as an effective inhibitor of angiogenesis and used to treat patients with HCC.
\end{abstract}

Correspondence to: Professor Xiaodong Gai, Department of Pathology, School of Basic Medical Sciences, Beihua University, 3999 Huashan Road, Jilin City, Jilin 132013, P.R. China

E-mail: bhdxgaixiaodong@126.com

Key words: tumor angiogenesis, tumstatin, hepatocellular carcinoma, retroviral vector

\section{Introduction}

Hepatocellular carcinoma (HCC), one of the most common types of cancer in the world, is a hypervascular carcinoma. Angiogenesis serves an important role in HCC progression, malignancy, metastasis and high rates of recurrence (1-3). Solid tumors may not grow bigger than $2-3 \mathrm{~mm}^{3}$ if tumor angiogenesis is blocked (4). Therefore, the development of genetic engineering technologies to target angiogenesis may be a novel and effective method of treating HCC.

Angiogenesis enables tumor growth and metastasis to occur. Moreover, it is the process by which tumor cells are provided with a supply of blood and nutrients (5-7). Due to its extensive role in inducing cancer cell growth, tumor angiogenesis has become a novel and promising target for anticancer therapy. Inhibiting tumor angiogenesis may be an efficient way of preventing tumor occurrence and progression.

Tumstatin is a novel factor that inhibits vascular endothelial cell growth. It belongs to the $\mathrm{NC} 1$ domain of $\alpha 3$ chain of type-IV collagen and exerts anti-angiogenic activity $(8,9)$. Tumstatin binds to endothelial cell surface integrins and exerts its effects through multiple mechanisms, including inhibition of endothelial cell protein synthesis, which results in endothelial cell apoptosis, inhibition of tumor blood vessel formation, and inhibition of tumor cell growth, invasion and metastasis (9). The anti-angiogenic activity of tumstatin is localized to its 54-132 amino acid region (Tum-5), which exhibits similar biological activity to the parent protein (10-12).

The aim of the present study was to evaluate whether gene therapy with Tum-5 is an effective strategy to treat patients with HCC. The Tum-5 gene fragment was cloned and inserted into a pLXSN retroviral vector following the protocol of a previous study by our group (13). To identify the role Tum-5 serves in the process of HCC growth, the anti-angiogenic and antitumor effects of pLXSN-Tum-5 virus transfection were assessed in vitro and in vivo.

\section{Materials and methods}

Reagents. The rabbit anti-mouse CD31 monoclonal antibody was purchased from eBioscience, Inc. (cat. no. 13-0311-81; San Diego, CA, USA). The Ready-to-Use Immunohistochemistry Hypersensitivity UltraSensitive ${ }^{\text {TM }}$ S-P kit was purchased from Maixin Biotech. Co., Ltd. (Fuzhou, China). MTT and 
Polybrene ${ }^{\circledR}$ were purchased from Sigma-Aldrich (Merck Millipore, Darmstadt, Germany).

Cells and culture. The retroviral packaging mouse fibroblast cell line PA317, NIH3T3 fibroblasts, human umbilical vein endothelial cells (HUVECs), the HepG2 human hepatocarcinoma cell line and the H22 mouse hepatocarcinoma cell line were all obtained from American Type Culture Collection (Manassas, VA, USA). Cells were cultured at $37^{\circ} \mathrm{C}$ with $5 \% \mathrm{CO}_{2}$ in complete Dulbecco's Modified Eagle's medium (Gibco; Thermo Fisher Scientific, Inc., Waltham, MA, USA) containing 10\% FBS (Gibco; Thermo Fisher Scientific, Inc.).

Production of viral particles and determination of viral titer. The human pLXSN-Tum-5 plasmid was constructed as previously described (13). A total of $5 \times 10^{5}$ PA317 packaging cells were seeded in 6-well plates. After 24 h, pLXSN-Tum-5 and pLXSN were transfected into the PA317 cells using Lipofectamine $^{\circledR} 2000$ (Invitrogen; Thermo Fisher Scientific, Inc.) for $6 \mathrm{~h}$. Infected cells were split and grown with $400 \mu \mathrm{g} / \mathrm{ml}$ G418-containing medium. After 2 weeks, G418-resistant colonies were picked, the virus-containing supernatant was collected and passed through a $0.45 \mu \mathrm{m}$ filter, subsequently frozen and stored at $-80^{\circ} \mathrm{C}$. The titer of viral stocks was determined by infection of the NIH3T3 cells using a previously described technique (14). The titers of pLXSN-Tum-5 and pLXSN virus were $8.2 \times 10^{6}$ colony-forming units $/ \mathrm{ml}$ and $7.6 \times 10^{6}$ colony-forming units $/ \mathrm{ml}$, respectively.

MTT assay for cell proliferation in vitro. Cell growth was evaluated using an MTT assay. HUVECs or HepG2 cells were seeded in 96 -well plates at $8 \times 10^{3}$ cells/well $(0.2 \mathrm{ml} /$ well $)$ and cultured at $37^{\circ} \mathrm{C}$ in $5 \% \mathrm{CO}_{2}$ overnight to allow for cell attachment. Cells were subsequently infected with pLXSN-Tum-5 virus at $0,1,5,10,25$ and 50 multiplicity of infection (MOI) in the presence of $5 \mu \mathrm{g} / \mathrm{ml}$ Polybrene. Following $72 \mathrm{~h}$ of incubation, the supernatant was removed and serum-free culture medium containing $5 \mathrm{mg} / \mathrm{ml}$ MTT was added to each well. Following $4 \mathrm{~h}$ of incubation with MTT, the supernatant was discarded and $150 \mu 1$ dimethyl sulfoxide (Sigma-Aldrich; Merck Millipore) was added for $10 \mathrm{~min}$. The optical density (OD) of each well was measured at $570 \mathrm{~nm}$ using a Bio-Rad 2550 microplate reader (Bio-Rad Laboratories, Inc., Hercules, CA, USA). All experiments were performed in triplicate.

Antitumor effects in vivo. The antitumor effects of retroviral vectors containing Tum-5 gene were examined in vivo using a H22 mouse HCC xenograft implanted in Kunming (KM) female mice (55-70 days old; weight, 15-20 g) that were obtained from Jilin University (Changchun, China). The mice were implanted subcutaneously with $1 \times 10^{6} \mathrm{H} 22$ cells in $0.1 \mathrm{ml}$ serum-free medium to produce a subcutaneous tumor xenograft. The mice were housed in sterile prebedded plastic cages and maintained at $20^{\circ} \mathrm{C}$ with a $12 \mathrm{~h} \mathrm{light} / 12 \mathrm{~h}$ dark cycle and had free access to mouse food and water. When the tumor size reached $30-70 \mathrm{~mm}^{3}, 15$ xenograft-bearing mice were randomly divided into three groups: Saline ( $\mathrm{n}=5)$, pLXSN (empty virus; $n=5)$ and pLXSN-Tum-5 $(n=5)$. Injections of saline, pLXSN and pLXSN-Tum-5 were administered on days $0,2,4,6$ and 8 into tumor tissues at a MOI of 5 per mouse. The tumor size and body weight of each mouse were recorded every other day. The antitumor effects were determined by measuring the tumor dimensions via vernier caliper to the nearest $0.1 \mathrm{~mm}$, and calculating the volume using the following equation: $\mathrm{V}=\mathrm{ab}^{2} / 2$, where $\mathrm{a}$ and $\mathrm{b}$ represent the length and width of tumor, respectively. After 10 days, mice under pentobarbital anesthesia ( $80 \mathrm{mg} / \mathrm{kg}$ body weight; Sigma-Aldrich) were sacrificed by cervical dislocation, and tumor tissues were carefully excised from the body and weighed. All animal experiments were performed in compliance with the NIH guidelines for the care and use of laboratory animals. The animal experiments in this study were approved by the Animal Ethics Committee of Beihua University (Jilin City, China).

Immunohistochemical staining for CD31. Tumor tissues from the H22 tumor-bearing mice (saline, pLXSN and pLXSN-Tum-5 groups) were fixed in 10\% formalin at room temperature for $24 \mathrm{~h}$, embedded in paraffin and cut into $4-\mu \mathrm{m}$ consecutive sections. Following deparaffinization and antigen retrieval, immunohistochemical staining was performed using the Ready-to-Use Immunohistochemistry Hypersensitivity UltraSensitive ${ }^{\mathrm{TM}}$ S-P kit according to the manufacturer's instructions. Sections were treated with $3 \%$ hydrogen peroxide for $10 \mathrm{~min}$ at room temperature to block the activity of endogenous peroxidase. The sections were washed with phosphate-buffered saline (PBS) for $5 \mathrm{~min}$ and blocked with normal goat serum (provided with the kit) for $10 \mathrm{~min}$ at room temperature. The sections were subsequently incubated with a 1:100 dilution of the monoclonal antibody for CD31 at $4^{\circ} \mathrm{C}$ overnight. The sections were then washed with PBS and treated with biotinylated secondary antibody (provided with the kit) for $10 \mathrm{~min}$, followed by further incubation with streptavidin-horseradish peroxidase complex. Following additional washing, diaminobenzidine was used as a chromogen and counterstaining was performed using hematoxylin. Sections were dehydrated, cleared and mounted with resin.

Microvessel density (MVD). From the CD31-stained sections, the MVD was determined at the hot spot through light microscopy examination (BX43F; Olympus Corporation, Tokyo, Japan). For each section, positively stained microvessels were counted from 5 high-power fields (HPF; magnification, x 400). The average count was regarded as the MVD per HPF.

Statistical analysis. Statistical analysis was performed with the Statistical Package for Social Sciences version 17.0 (SPSS, Inc., Chicago, IL, USA). All experiments were performed at least three times and all values were expressed as the mean \pm standard deviation. Student's $t$-test was used to compare values between two groups and one-way analysis of variance (ANOVA) was used for more than two groups. For all analyses, $\mathrm{P}<0.05$ was considered to indicate a statistically significant difference.

\section{Results}

Effect of Tum-5 transfection on HUVEC growth in vitro. To examine the role of Tum-5 in tumor angiogenesis, HUVECs were used to mimic the tumor environment. HUVECs were transfected with pLXSN-Tum-5 or pLXSN virus at different 

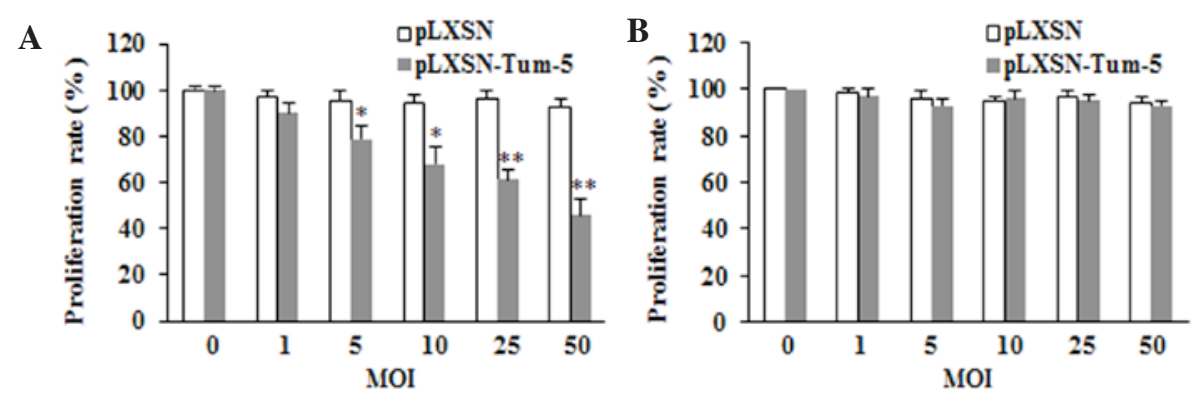

Figure 1. Effect of Tum-5 on cell growth in vitro. The proliferation rate of (A) human umbilical vein endothelial cells and (B) HepG2 cells was assessed following pLXSN-Tum-5 and pLXSN virus transfection at MOIs of $0,1,5,10,25$ and 50 for $72 \mathrm{~h}$. ${ }^{*} \mathrm{P}<0.05$ and ${ }^{* *} \mathrm{P}<0.01$, compared with the pLXSN group. Tum, tumstatin; MOI, multiplicity of infection.
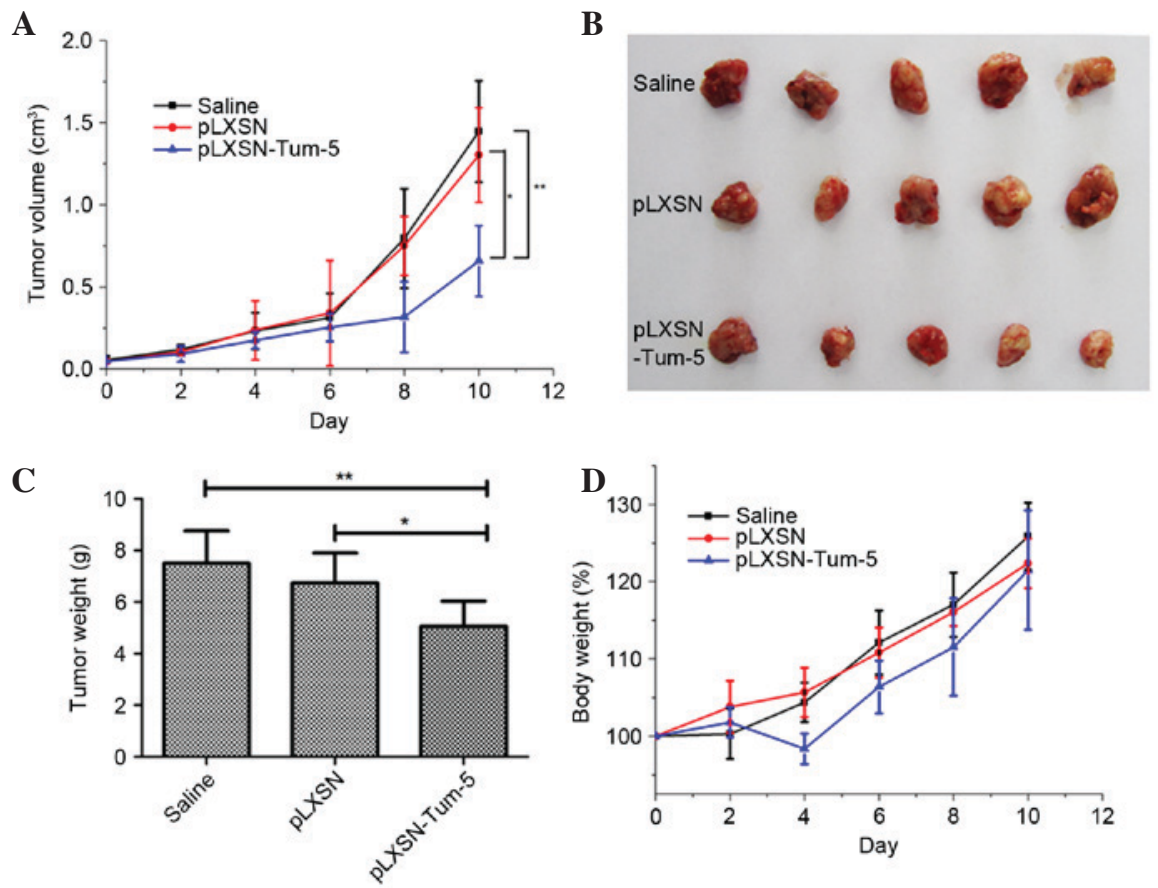

Figure 2. Antitumor efficacy of Tum-5 in vivo. (A) Tumor volume of H22 tumor-bearing mice treated with saline, pLXSN and pLXSN-Tum-5 measured over 10 days. Mice received injections on days $0,2,4,6$ and 8 . (B) Images of excised tumors on day 10. (C) Weight of excised tumors. (D) Body weight of H22 tumor-bearing mice treated with saline, pLXSN and pLXSN-Tum- 5 over 10 days. ${ }^{*} \mathrm{P}<0.05$ for the pLXSN-Tum-5 group vs. the pLXSN group and ${ }^{* *} \mathrm{P}<0.01$ for the pLXSN-Tum-5 group vs. the saline group. Tum, tumstatin.

MOIs of $0,1,5,10,25$ and 50 . The proliferation rate was measured using the MTT assay at $72 \mathrm{~h}$. The results demonstrated that cells in the pLXSN-Tum-5 group exhibited a significantly lower proliferation rate compared with the pLXSN group $(\mathrm{P}<0.05)$. Tum-5 significantly inhibited the proliferation of HUVECs in a titer-dependent manner (Fig. 1A).

Effect of Tum-5 transfection on HepG2 cell growth in vitro. Based on the anti-angiogenesis activity of Tum-5 in HUVECs, it was investigated whether Tum-5 exerts antitumor activity in tumor cells in vitro. HepG2 cells were transfected with Tum-5 virus at different MOIs of $0,1,5,10,25$ and 50 for $72 \mathrm{~h}$. The results demonstrated that the growth of the pLXSN-Tum-5 group was not significantly different from that of the pLXSN group ( $\mathrm{P}>0.05$; Fig. 1B). This result indicated that the introduction of Tum-5 into HepG2 cells did not affect HepG2 cell proliferation.
Antitumor effect of Tum-5 in vivo. To test if the retrovirus containing the Tum-5 gene was able to inhibit tumor growth in vivo, a $\mathrm{H} 22$ mouse HCC xenograft model was employed. When the tumor size reached $30-70 \mathrm{~mm}^{3}$, the 15 mice were randomly divided into three equal groups. Tumors were injected with pLXSN or pLXSN-Tum-5 retroviral vectors (MOI of 5 per mouse) on days $0,2,4,6$ and 8 . The results indicated that, compared with saline $(\mathrm{P}<0.01)$ and empty retroviral vectors $(\mathrm{P}<0.05)$, pLXSN-Tum-5 significantly inhibited tumor growth on day 10, after the administration of 5 injections (Fig. 2A). Considering that pLXSN had no effect on tumor growth, it was concluded that tumor growth was inhibited by the Tum- 5 gene carried by the retroviral vector. Tumor tissues were excised on the 10th day and weighed. The size and weight of tumors from mice in the pLXSN-Tum-5 group were significantly lower than those of mice in the saline $(\mathrm{P}<0.01)$ and $\mathrm{pLXSN}$ groups $(\mathrm{P}<0.05)$ (Fig. $2 \mathrm{~B}$ and $\mathrm{C})$. 
A

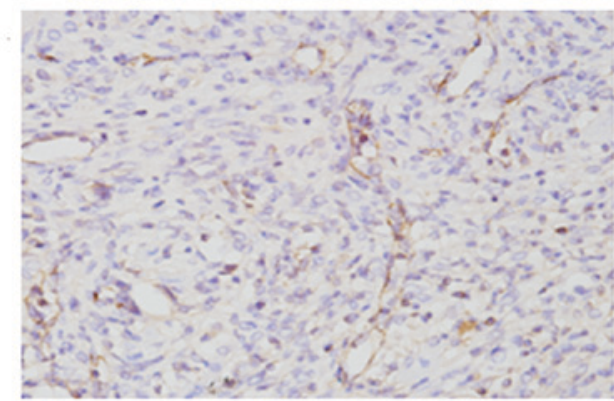

C

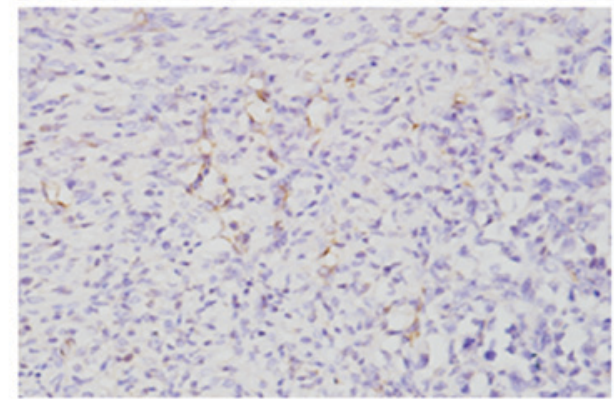

B

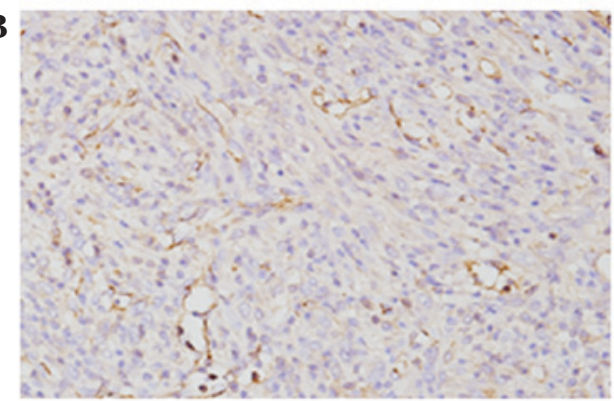

D

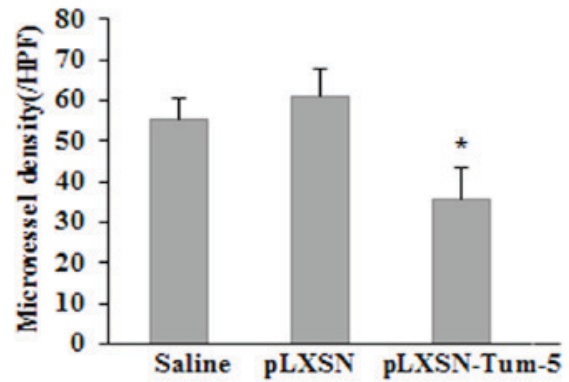

Figure 3. Immunostaining for CD31 in tumor tissues from H22 xenograft mice in (A) the saline group, (B) the pLXSN group and (C) the pLXSN-Tum-5 group (magnification, $\mathrm{x} 400$ ). (D) Graph of average microvessel density in CD31-stained sections. * $\mathrm{P}<0.05$ compared with saline and pLXSN groups. HPF, high-power field; Tum, tumstatin.

Changes in body weight were used to evaluate the toxicity of the retroviral vectors. The results demonstrated no significant weight loss in mice in the pLXSN-Tum5 group compared with mice from the other groups following 10 days of treatment, suggesting that retroviral vectors containing the Tum-5 gene exhibit low toxicity (Fig. 2D). The notable weight loss of the pLXSN-Tum-5 group at day 4 may be attributed to the inhibited tumor growth caused by Tum-5.

Expression of CD31 in tumor tissue. As no direct antitumor effect of Tum-5 was detected in vitro, it was speculated that the antitumor effect of Tum-5 observed in vivo was ascribed to the failure of angiogenesis in H22 tumor-bearing mice. Therefore, the endothelial cell marker CD31, was used to analyze tumor angiogenesis. Tumor tissues from H22 tumor-bearing mice were immunohistochemically stained with an antibody against CD31. The average MVD of CD31-stained sections in the pLXSN-Tum-5 group was significantly lower than that in the saline and pLXSN groups $(\mathrm{P}<0.05$; Fig. 3$)$. These results suggested that Tum-5 may decrease HCC tumor growth by inhibiting angiogenesis in vivo.

\section{Discussion}

Tumor angiogenesis is a key process for the majority of solid tumors, since the growth and metastasis of malignant cells require the formation of new blood vessels $(5-7,15,16)$. Tumstatin specifically inhibits endothelial cell proliferation and induces apoptosis by interacting with $\alpha_{\mathrm{V}} \beta_{3}$ integrin $(12,17)$. Deletion mutagenesis analysis has revealed that the anti-angiogenic activity of tumstatin is localized to the 54-132 amino acid region (Tum-5) (11). It is easier to transfer smaller fragments into tissue or cells, and in the present study, Tum-5 cDNA was therefore transfected into the retrovirus plasmid pLXSN to construct pLXSN-Tum-5 (13), a carrier which can effectively transfect host cells to stably express Tum-5 (18). To determine whether Tum-5 exhibits anti-angiogenic activity, HUVECs were transfected with pLXSN-Tum-5 virus in vitro. The results of the MTT assay indicated that the transfection of pLXSN-Tum-5 virus significantly decreased HUVEC proliferation in a titer-dependent manner and that the Tum-5 fragment, like full-length tumstatin, exhibits proliferation-inhibitory activity.

It has been demonstrated that tumstatin inhibits tumor growth in a number of different types of cancer, including human renal carcinoma, prostate carcinoma, lung carcinoma and glioma $(11,12,19-22)$. As a gene fragment encoding the region of tumstatin with anti-angiogenic activity, Tum-5 has been identified to exhibit antitumor activity by exerting anti-angiogenic activity, but does not act directly on tumor cells (10-12). However, it has been reported that the Tum-5 gene may significantly inhibit gastric cancer cell proliferation and promote cell apoptosis in vitro (23). Therefore, the present study evaluated the antitumor effects of Tum-5 on HepG 2 cells in vitro. The results showed that the proliferation of HepG2 cells transfected with Tum-5 did not significantly differ from that of cells transfected with retroviral vector, suggesting that Tum-5 had no direct antitumor activity on HepG2 cells. It was considered that Tum-5 may have different effects on gastric cancer and HCC cells. However, Tum-5 did not exert an antitumor effect on $\mathrm{H} 22$ cells in vitro in our prelimary experiment. In blood vessels formed during tumor angiogenesis, vascular endothelial cells may serve important roles in several steps of tumor cell activation, proliferation, migration, invasion and tubule formation $(24,25)$. Considering the rich blood vessels observed in $\mathrm{HCC}$, specific angiogenesis-targeting interventions provide a possible treatment for $\mathrm{HCC}$ by inhibiting the abovementioned processes (26-28). Therefore, the inhibition of angiogenesis represents a potential therapeutic target for $\mathrm{HCC}$ that may reduce the mortality of patients with HCC. The 
present study investigated the inhibitory effects of Tum-5 on HCC growth in vivo by injecting pLXSN-Tum-5 virus into KM mice bearing $\mathrm{H} 22$ tumors, and a significant antitumor effect was observed. The size and weight of tumors from mice in the pLXSN-Tum-5 group were significantly lower than those from the pLXSN and wild-type $\mathrm{H} 22$ HCC groups, suggesting that Tum-5 is effective at inhibiting HCC tumor growth.

The rate of angiogenesis is typically estimated by measuring the MVD of fixed tissue immunostained for endothelial markers, including CD31, CD34 and factor VIII. CD31 is a pan-endothelial marker for small and large vessels (29). In order to assess the MVD, CD31 and associated antibodies are used to mark tumor vascular endothelial cells and the capillary number per unit area is counted (30). In the present study, immunohistochemical staining for CD31 revealed that the average MVD in the pLXSN-Tum-5 group was significantly lower compared with that in the pLXSN and wild-type H22 HCC groups. The results indicated that Tum-5 may have a significant anti-angiogenic effect on the neovascular endothelial cells of HCC.

In conclusion, the present study determined that Tum-5 specifically inhibited HUVEC proliferation, but did not have any direct effect on $\mathrm{HCC}$ cell growth in vitro. The in vivo study demonstrated that Tum-5 exerted stronger antitumor activity through suppression of vascular endothelial cells compared with direct action on HCC cells. Taken together, the results of the present study suggested that the gene fragment of Tum-5 may be an effective angiogenesis inhibitor and may be developed as novel therapeutic strategy to treat patients with HCC.

\section{Acknowledgements}

The present study was supported by the Science and Technology Department of Jilin province (grant nos. 20110728 and 20150101128JC) and by the Health Department of Jilin Province (grant no. 20122120).

\section{References}

1. Yuan MM, Xu YY, Chen L, Li XY, Qin J and Shen Y: TLR3 expression correlates with apoptosis, proliferation and angiogenesis in hepatocellular carcinoma and predicts prognosis. BMC Cancer 15: 245, 2015.

2. Lin W, Zhao J, Cao Z, Zhuang Q, Zheng L, Zeng J, Hong Z and Peng J: Livistona chinensis seeds inhibit hepatocellular carcinoma angiogenesis in vivo via suppression of the Notch pathway. Oncol Rep 31: 1723-1728, 2014

3. Wang W, Li GY, Zhu JY, Huang DB, Zhou HC, Zhong W and Ji CS: Overexpression of AGGF1 is correlated with angiogenesis and poor prognosis of hepatocellular carcinoma. Med Oncol 32: $131,2015$.

4. Lewis CE, Leek R, Harris A and McGee JO: Cytokine regulation of angiogenesis in breast cancer: The role of tumor-associated macrophages. J Leukoc Biol 57: 747-751, 1995.

5. Folkman J: Tumor angiogenesis: Therapeutic implications. N Engl J Med 285: 1182-1186, 1971.

6. Folkman J: Angiogenesis in cancer, vascular, rheumatoid and other disease. Nat Med 1: 27-31, 1995

7. Bielenberg DR and Zetter BR: The contribution of angiogenesis to the process of metastasis. Cancer J 21: 267-273, 2015.

8. Hamano Y, Zeisberg M, Sugimoto H, Lively JC, Maeshima Y, Yang C, Hynes RO, Werb Z, Sudhakar A and Kalluri R: Physiological levels of tumstatin, a fragment of collagen IV alpha3 chain, are generated by MMP-9 proteolysis and suppress angiogenesis via alphaV beta3 integrin. Cancer Cell 3: 589-601, 2003 .
9. Sudhakar A and Boosani CS: Inhibition of tumor angiogenesis by tumstatin: Insights into signaling mechanisms and implications in cancer regression. Pharm Res 25: 2731-2739, 2008.

10. Maeshima Y, Manfredi M, Reimer C, Holthaus KA, Hopfer H, Chandamuri BR, Kharbanda S and Kalluri R: Identification of the anti-angiogenic site within vascular basement membrane-derived tumstatin. J Biol Chem 276: 15240-15248, 2001.

11. Maeshima Y, Colorado PC, Torre A, Holthaus KA, Grunkemeyer JA, Ericksen MB, Hopfer H, Xiao Y, Stillman IE and Kalluri R: Distinct antitumor properties of a type IV collagen domain derived from basement membrane. J Biol Chem 275: 21340-21348, 2000.

12. Maeshima Y, Colorado PC and Kalluri R: Two RGD-indepentdent alpha vbeta3 integrin binding sites on tumstatin regulate distinct anti-tumor properties. J Biol Chem 275: 23745-23750, 2000.

13. Gai XD, Luo H, Li C and Feng K: The construction of recombined retroviral plasmid with human Tum-5 gene and packing cell line. Zhouguo Lao Nian Xue Zazhi 29: 1194-1196, 2009 (In Chinese).

14. Bodine DM, McDonagh KT, Brandt SJ, Ney PA, Agricola B, Byrne E and Nienhuis AW: Development of a high-titer retrovirus producer cell line capable of gene transfer into rhesus monkey hematopoietic stem cells. Proc Natl Acad Sci USA 87: 3738-3742, 1990 .

15. Bisacchi D, Benelli R, Vanzetto C, Ferrari N, Tosetti F and Albini A: Anti-angiogenesis and angioprevention: Mechanisms, problems and perspectives. Cancer Detect Prev 27: 229-238, 2003.

16. Chen Y, Gou X, Ke X, Cui H and Chen Z: Human tumor cells induce angiogenesis through positive feedback between CD147 and insulin-like growth factor-I. PLoS One 7: e40965, 2012.

17. Pedchenko V,Zent R and Hudson BG: Alpha(v)beta3 and alpha(v) beta5 integrins bind both the proximal RGD site and non-RGD motifs within noncollagenous ( $\mathrm{NC1}$ ) domain of the alpha3 chain of type IV collagen: Implication for the mechanism of endothelia cell adhesion. J Biol Chem 279: 2772-2780, 2004.

18. McTaggart S and Al-Rubeai M: Retroviral vectors for human gene delivery. Biotechnol Adv 20: 1-31, 2002.

19. Maeshima Y, Yerramalla UL, Dhanabal M, Holthaus KA, Barbashov S, Kharbanda S, Reimer C, Manfredi M, Dickerson WM and Kalluri R: Extracellular matrix-derived peptide binds to alpha(v)beta(3) integrin and inhibits angiogenesis. J Biol Chem 276: 31959-31968, 2001.

20. Sund M, Hamano Y, Sugimoto H, Sudhakar A, Soubasakos M, Yerramalla U, Benjamin LE, Lawler J, Kieran M, Shah A and Kalluri R: Function of endogenous inhibitors of angiogenesis as endothelium-specific tumor suppressors. Proc Natl Acad Sci USA 102: 2934-2939, 2005.

21. Ye HX, Yao Y, Jiang XJ and Yuan XR: Tumstatin transfected into human glioma cell line U251 represses tumor growth by inhibiting angiogenesis. Chin Med J (Engl) 126: 1720-1725, 2013.

22. You Y, Xue X, Li M, Qin X, Zhang C, Wang W, Giang C, Wu S, Liu Y, Zhu W, et al: Inhibition effect of pcDNA-tum-5 on the growth of S180 tumor. Cytotechnology 56: 97-104, 2008.

23. Zhang J: A study on the influence of proliferation and apoptosis for Tum-5 gene to human gastric carcinoma cell. Masters thesis, Fujian Medical University, Fuzhou, China, 2010 (In Chinese).

24. Jahroudi $\mathrm{N}$ and Greenberger JS: The role of endothelial cells in tumor invasion and metastasis. J Neurooncol 23: 99-108, 1995.

25. Wang YH, Dong YY, Wang WM, Xie XY, Wang ZM, Chen RX, Chen J, Gao DM, Cui JF and Ren ZG: Vascular endothelial cells facilitated HCC invasion and metastasis through the Akt and $\mathrm{NF}-\kappa \mathrm{B}$ pathways induced by paracrine cytokines. J Exp Clin Cancer Res 32: 51, 2013.

26. Zhang ZL, Zhang JF, Yuan YF, He YM, Liu QY, Mao XW, Ai YB and Liu ZS: Suppression of angiogenesis and tumor growth in vitro and in vivo using an anti-angiopoietin-2 single-chain antibody. Exp Ther Med 7: 543-552, 2014.

27. Grothey A and Galanis E: Targeting angiogenesis: Progress with anti-VEGF treatment with large molecules. Nat Rev Clin Oncol 6: 507-518, 2009.

28. Sugimachi K, Tanaka S, Taguchi K, Aishima S, Shimada M and Tsuneyoshi M: Angiopoietin switching regulates angiogenesis and progression of human hepatocellular carcinoma. J Clin Pathol 56: 854-860, 2003.

29. Hasan J, Byers R and Jayson GC: Intra-tumoural microvessel density in human solid tumours. Br J Cancer 86: 1566-1577, 2002.

30. Cârţână T, Săftoiu A, Gruionu LG, Gheonea DI, Pirici D, Georgescu CV, Ciocâlteu A and Gruionu G: Confocal laser endomicroscopy for the morphometric evaluation of microvessels in human colorectal cancer using targeted anti-CD31 antibodies. PLoS One 7: e52815, 2012. 